\section{Lymphedema is more than excess of fluid; a lympho-fibro-adipo-edema}

\section{Waldemar L. Olszewski, Marzanna Zaleska, Marta Cakala}

Central Clinical Hospital, Dept. of General and Vascular Surgery and Mossakowski Medical Research Center, Dept. of Applied Physiology, Polish Academy of Sciences, Warsaw, Poland

\section{Introduction}

Lymphedema has been defined as an abnormal accumulation of capillary filtrate with proteins, cytokines and chemokines, recirculating lymphocytes, products of parenchymatous cells, and debris of senescent cells caused by obstruction of the main lymphatics following bacterial inflammation, mechanical trauma, excision of lymph nodes with afferent lymphatics and local irradiation in cancer therapy. ${ }^{1}$ Moreover, stagnant fluid is colonized by microorganisms penetrating foot or hand skin clinically diagnosed as dermato-lymphangio-adenitis (DLA) (previously called cellulitis). ${ }^{2}$ All substances and elements accumulating in the intercellular space change the residential cells' environment. This subsequently brings about structural and functional changes in the tissues and limbs.

Tissue specimens obtained during debulking surgery (Figure 1) as well as MRI (magnetic resonance imaging) (Figure 2) and US (ultrasonography) pictures show that fluid is only one element in increased limb volume. There is increase of fibrous tissue mass, formation of multiple fibrous septa encompassing multiple fat globules in whole limb. Fibrotic and adipose tissue increase limb volume and disable limb function. These observations ponder upon the results of compression of swollen tissues. The externally applied force deforms tissues and dissipates according to the size of the surface area of the compressed limb fragment. It is absorbed in the epidermis by keratinocytes, basal membrane, blood capillaries, blood and lymph in the subepidermal plexus and in dermis by elastic and collagen fibers, fibroblasts and adipocytes, blood vessels filled with blood under pressure, and interstitial fluid present between cells and fibers (Figure 3). ${ }^{3}$ Moreover, the gross tissue structure and fluid content differ at various limb levels depending on the volume of soft tissue and accumulated mobile edema fluid.

\section{Aim}

To study topography of edema fluid accumulation and fibrous and fat tissue mass.

\section{Materials and Methods}

Twenty patients with lymphedema of lower limbs stage III and IV, proved of lymphoscintigraphy, were studied. Biopsy specimens were collected from leg above the
Correspondence: Waldemar L. Olszewski, Central Clinical Hospital, Dept. of General and Vascular Surgery and Mossakowski Medical Research Center, Dept. of Applied Physiology, Polish

Academy of Sciences, Warsaw, Poland.

E-mail: waldemar.1.olszewski@gmail.com

Conference presentation: International Compression Club (ICC) Meeting, Rotterdam, 2018.

This work is licensed under a Creative Commons Attribution 4.0 License (by-nc 4.0).

(C) Copyright W.L. Olszewski et al., 2018

Licensee PAGEPress, Italy

Veins and Lymphatics 2018; 7:7984

doi:10.4081/vl.2018.7984

ankle joint. Specimens stained with hematoxylin-eosin, trichrome and Paris Blue in chloroform and glycerol for transparency. Area of specifically stained elements calculated under microscope with Microimage program (Olympus, Japan).

\section{Results}

Hyperkeratosis (5-7 keratinocyte layers) (Figure 4). A 3-7 mm thick dermis layer. Subcutaneous tissue with a honeycomb structure and fibrous septa of different thickness. Adipose tissue 20-30\% (Figure 5) and fibrous elements $30 \%$ of specimen area, $15-40 \%$ spaces filled with fluid (Figure 6).

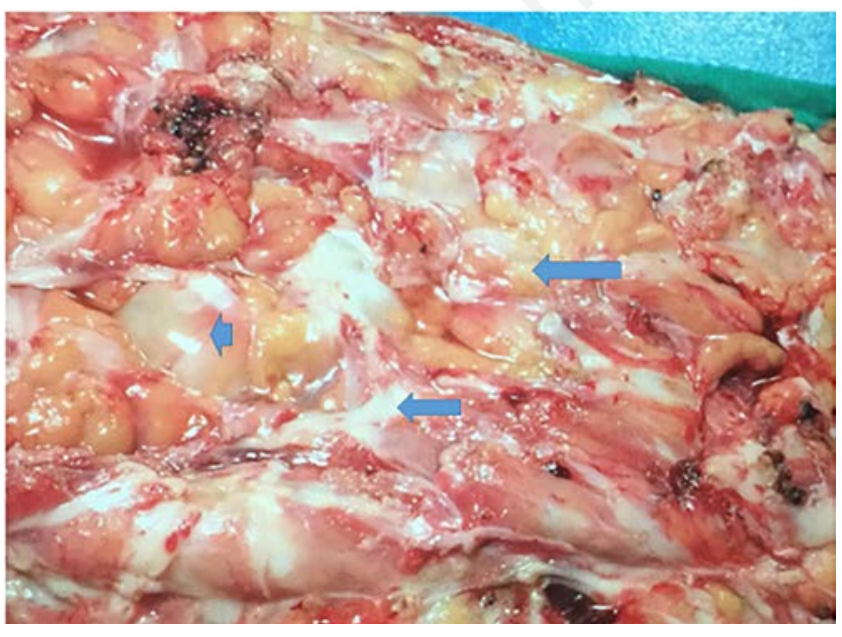

Figure 1. Skin and subcutaneous tissue specimen obtained during debulking surgery in lower limb lymphedema stage III. The tickness is $6 \mathrm{~cm}$, large fat tissue globules (large arrow) separated by fibrous septa (smaller arrow) and fluid covering the surface are seen. A fluid blister is seen between solid structures (arrowhead).

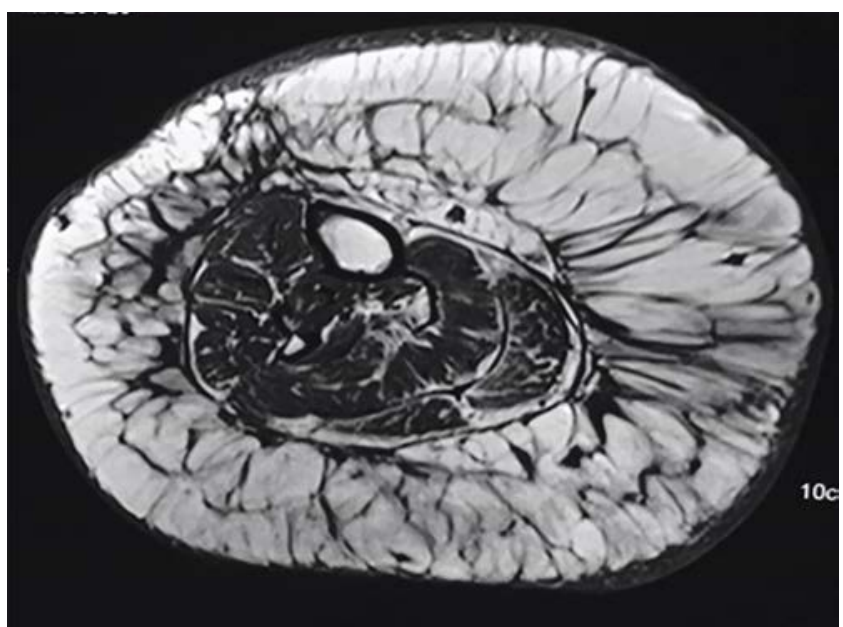

Figure 2. MRI picture of lymphedematous thigh showing thick skin, expanded subcutaneous compartment with fat globules separated by fibrous septa and fluid accumulating in all free spaces. This picture corresponds to that of the harvested skin and subcutis fragment presented on Figure 2. 


\section{Discussion and Conclusions}

Lymphedema of lower limbs in advanced stage is an increase in limb volume caused by accumulation of: i) fluid; and ii) cell and matrix mass. Excess capillary filtrate/tissue fluid locates in tissue spaces, but not lymphatics, as they are not patent (except of the subdermal plexus, formerly dermal backflow, $<5 \%$ of total lymphatic space). In addition, there is deposi- tion of collagen and hypertrophy and later also hyperplasia of adipocytes. Inflammatory processes (DLA) complicating lymph stasis further contribute to volume gain by increased capillary permeability and filtration of plasma fluid.

Translating it into the plain clinical language, increase in limb volume in lymphatic pathways obstruction is a sum of increased mobile fluid and cellular mass volume. This is not exactly known by patients and therapists who expect a visible effect of compression therapy in terms of decreased fluid volume ${ }^{4}$ but increase in tissue elasticity (decrease of stiffness). ${ }^{5}$ Structure of the subcutaneous tissue of the edematous limb is like that of a sponge. The tissue skeleton is built mostly of collagen. Fluid accumulates between the collagen bundles. During massage, fluid is moved away but collagen fibers recoil force remains unchanged. Therefore, after mas-

\section{Compression sleeve}

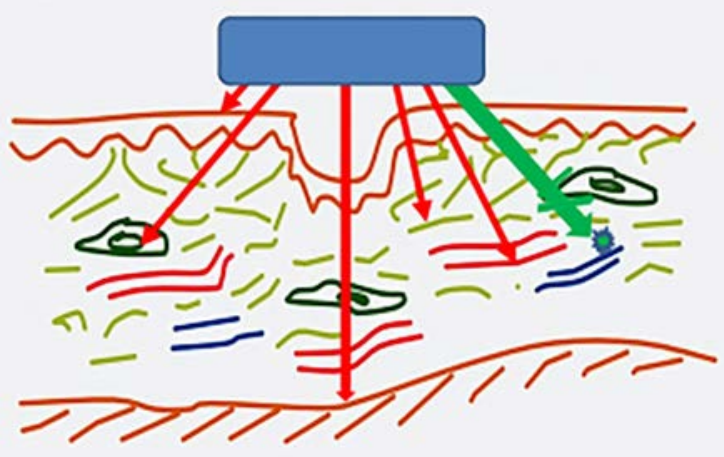

Figure 3. Schematic presentation of tissue structures absorbing compression force of a compression sleeve. These are epidermis, subcutaneous tissue fibroblasts, muscular fascia, collagen and elastic fibers, blood vessels (red arrows). The remaining force moves tissue (edema) fluid (green arrow) when solid structures become deformed.

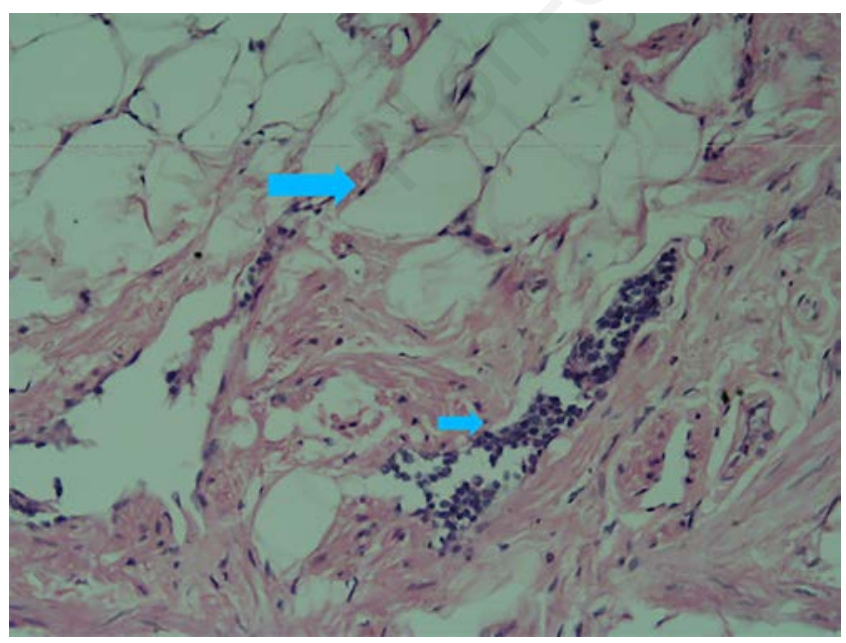

Figure 5. Histological picture of fat tissue with hyperplastic adipocytes (large arrow) in leg lymphedema stage III. Interestingly, focal mononuclear aggregates (small arrow) in dilated lymphatics. Fat cells, fibrosis and fluid in tissue spaces create a typical picture in advanced stages of lymphedema. H-E stain, magnification $\mathrm{x} 40$.

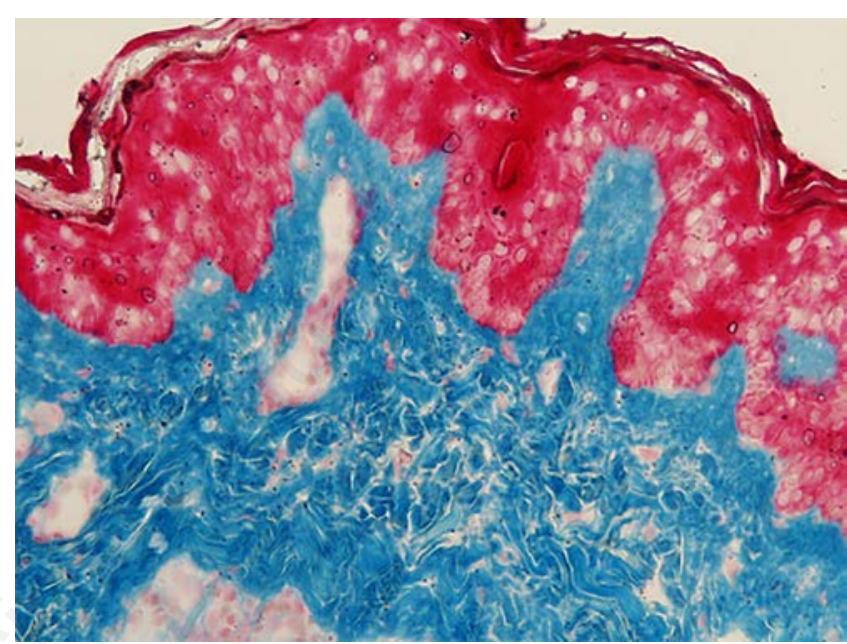

Figure 4. Histological specimen of skin from a lymphedema calf stage III. Hyperkeratosis-multiple keratinocyte layers, confluent areas of keratinocyte degeneration (red). Below dense accumulation of collagen with small capillary structures. Trichrome stain, magnification $\times 40$.

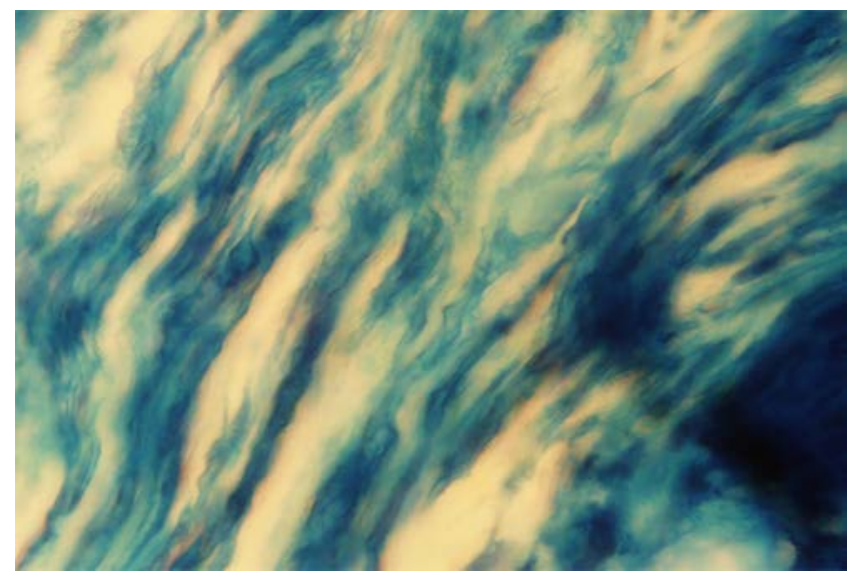

Figure 6. Specimen of fibrous tissue from a lymphedematous calf stage III depicting collagen fiber bundles separated by fluid. Around $50 \%$ of tissue occupied by fluid. Staining: Paris Blue in chloroform, transparency in glycerol, magnification $\mathbf{x} 40$. 
phatic system and its disorders. In: BB Lee, J Bergan, SG Rockson, ed. sage tissue skeleton regains its pre-massag shape, although there is less edema fluid. The limb circumference/volume is only slightly decreased but the sponge is $d r y$. Post-massage bandaging is then indispensable to prevent filling up by the newly formed capillary filtrate/tissue fluid.

\section{References}

1. Olszewski WL. Anatomy of the lym-
Lymphedema. Berlin: Springer; 2011. pp. 49-59.

2. Olszewski WL. In: BB Lee, J Bergan, SG Rockson, ed. Lymphedema. Berlin: Springer; 2011. pp. 207-211.

3. Olszewski WL, Jain P, Ambujam G, et al. Tissue fluid pressure and flow during pneumatic compression in lymphedema of lower limbs. Lymphat Res Biol 2011;9:77-83.

4. Zaleska M, Olszewski WL, Durlik M.
The effectiveness of intermittent pneumatic compression in long-term therapy of lymphedema of lower limbs. Lymphat Res Biol 2014;12:103-9.

5. Zaleska M, Olszewski WL, Durlik M, et al. Tonometry of deep tissues for setting effective compression pressures in lymphedema of limbs. Lymphat Res Biol 2017 [Epub ahead of print]. 